\title{
La ética como puerta a la informática en la educación primaria
}

\section{EthicsasaGateway to Computer ScienceinPrimaryEducation}

\author{
Juan Vicente Oltra Gutiérrez 1, Fernando José Garrigos Simon 1, Sofía Estelles Miquel 2
}

1 Escuela Técnica Superior de Ingeniería Informática (ETSINF) - Universitat Politècnica de València, España. jvoltra@omp.upv.es, fgarrigos@ doe.upv.es

2Facultad de Administración y Dirección de Empresas (FADE) - Universitat Politècnica de València, España. soesmi@omp.upv.es

\section{Resumen}

En el presente artículo se presenta una propuesta de acercamiento de la ética y la informática a estudiantes de los primeros cursos de primaria, apoyándose una en la otra, siguiendo el cauce del Real Decreto 126/2014, de 28 de febrero, por el que se establece el currículo básico de la Educación Primaria. Dentro del decreto se establecen como dos de las siete competencias del currículo la competencia digital (la tercera) y también las competencias sociales y cívicas (la quinta). Dada la población de nativos digitales que están recibiendo la formación, resultaría una meta un poco más ambiciosa el poder entreverarlas para que se apoyen la una a la otra. Dentro de este ámbito, por ejemplo, nos encontramos con alguna asignatura específica como "Valores sociales y cívicos" con criterios de evaluación tales como "Emplear las nuevas tecnologías desarrollando valores sociales y cívicos en entornos seguros". Gracias a esta puerta de entrada se pueden introducir pequeñas puertas a la visión de la informática, a través de la ética, que pueden ser transversales a la totalidad de las asignaturas del currículo. La sugerencia del presente artículo pasa por enfrentar a los discentes a una visión de la tecnología desde fuera de la tecnología, desde su prisma ético, una vez esta se apaga, y la pantalla de sus móviles o tabletas quedan convertidos en un mero espejo negro.

\section{Palabras Clave}

Enseñanza primaria; Ética; TIC; niños y adolescentes

\section{Abstract}

This paper presents a proposal to bring ethics and ICT closer to students of the first courses of the primary education, supporting one in each other, following the Law "Real Decreto 126/2014, 28th of February", which establishes the basic curriculum for Primary Education. Within this Law, two of seven skills in the curriculum are established: digital skill (the third) and also social and civic skills (the fifth). Given the digital natives population who are receiving education, it would be a slightly more ambitious goal to be able to glimpse them to support one in another. In this area, for example, we find a specific subject such as "Social and Civic values" with evaluation criteria such as "Employ new technologies by developing social and civic values in safe environments". Thanks to this gateway, we can introduce small door to the vision of computer science, through ethics, which may be transversal with all subjects of the curriculum. The suggestion of the present article is to confront teachers with a vision of technology from an outside perspective, from an ethical prism, once the technology is turned it off and the mobiles or tablets screens are converted into a mere black mirror.

\section{Keywords}

Primary education; Ethics; computer science; children and adolescents 


\section{Introducción}

Todo docente, todo ser humano que sienta su infancia más próxima al Equipo $A$ que a Dora la exploradora, sabe que hay un verdadero abismo entre su pasado y el presente de los niños actuales. Esta verdad de Perogrullo, que ya ha sido explorada por Prensky (2001) ha cambiado el modo de acercarse a los niños desde empresas (Oltra, 2014) a las propias instituciones, pasando por el múltiple y variado campo de la docencia.

La irrupción de las Tecnologías de la Información y las Comunicaciones (en adelante TIC) ha provocado una cadena de terremotos en todos los niveles de la enseñanza, desde preescolar a la universidad. No solo aparecen nuevas oportunidades, sino que los riesgos se presentan de forma a veces imperceptible, lo que los hace más peligrosos. Esto está presente en el día a día de nuestra sociedad, pero de forma gruesa: al analizar su efecto en la sociedad prima lo apologético, no solo a través de la prensa, sino también de nuestros políticos e incluso en ocasiones desde la universidad. Se vende la idea del horror más el error, focalizando en el caso de los niños además de en los peligros exteriores una sospecha de aislamiento, que provoca que no salgan de casa, obviando ventajas evidentes como que se les abre el acceso a las mayores bibliotecas del mundo.

Esta preocupación no es nueva. La irrupción temprana de la primera herramienta de comunicación global, la radio, ya trajo asociadas dudas como las actuales; así, apoyándonos en trabajo de Bringué y Sádaba (2009), vemos que en la década de los 30 del siglo XX una asociación de padres de la ciudad de Nueva York acusó a un programa de radio, Ether Bogeyman, de provocar pesadillas a sus hijos. Esto se reprodujo en la década de los 50 con la televisión y es claro precedente de temor al riesgo que las comunicaciones puedan tener sobre niños y adolescentes, sobre todo en lo referido al contenido y la conducta. Sin embargo, desde muy temprano es considerado de alto interés conseguir esa mezcla entreverada entre educación y TIC (Busnell, 1967) intentando ponderar cualidades y limitaciones, con tímidas reformas del currículo escolar.

Vivimos una simplificación más cercana a ser una ceremonia prelógica, un conjuro pseudo-religioso, antes que pensamiento crítico, pero no estamos ante un fenómeno tan nuevo como parece: hace décadas que el impacto de la informática en los individuos ha venido preocupando a pensadores y políticos. Desde el ya lejano Informe Nora Minc (Nora \& Minc, 1980) que en 1976 Giscard D'Estaing encargara a Simón Nora, Inspector General de Finanzas, como un informe sobre el desarrollo de las aplicaciones de la informática como factor de transformación económico y social y así "poder promoverla y a la vez dominarla", empieza a plantearse que la relación del ser humano con la informática podría llegar a cambiar profundamente al mismo. De esa fecha datan los trabajos de 
Weinzenbaum (1978) y pronto empieza a preocupar dentro de nuestras fronteras. Así, Campos (1985) lanza su discrepancia sobre la idea de una introducción de la informática en la educación periférica y superficial.

Actualmente, con el cambio acelerado de las TIC, coincidimos con Guerrero Cárdenas (2016) al señalar a la educación como el sector más afectado por el efecto que estas han producido, por una sencilla razón: los programas y los sistemas de enseñanza oficiales no solo no suelen estar a la par del progreso científico sino que, en ocasiones, obedecen a esquemas obsoletos.

Existen, claro está, numerosos antecedentes con experiencias que han luchado contra ese estado de cosas, de forma muy diversa, como el uso de videojuegos para inculcar valores (Moreno, 2016), trabajos sobre competencias digitales (Reche, 2016) o estrategias de "arriba abajo", preparando a los futuros docentes (Rayón, 2015). Cambia la educación porque cambia todo: no solo cambia la infancia, sino que es la misma sociedad la que da un giro radical. Cambios que no solo precisan de una especial atención en los primeros momentos de la formación intelectual de la persona en tanto en cuanto deben aprender a hacer de ese su entorno un medio seguro y confortable, sino además poder adaptarse a futuros cambios, incluso de difícil pronóstico hoy en día, propiciados por los avances en las tecnologías de la información y las comunicaciones.

Cabe citar un par de proyectos interesantes que guardan relación con el presente trabajo. Por un lado, el proyecto TACCLE 3 - Coding, que se encarga de introducir la programación y el pensamiento computacional en los niños de primaria (García-Peñalvo, 2016; García-Peñalvo, Reimann, Tuul, Rees \& Jormanainen, 2016), donde encontramos que una de sus actividades propuestas está muy relacionada con este trabajo (Seoane, 2016). El otro proyecto que está relacionado es el H2020 WYRED por su estudio de la influencia de la dimensión tecnológica en los jóvenes (García-Peñalvo \& Kearney, 2016).

\section{Contexto}

Decíamos en la introducción que la sociedad sufre una serie de cambios provocados por las tecnologías. Efectivamente, si el siglo XX fue el del gran salto tecnológico, que produjo fenómenos tales como que el mismo ser humano que pudo ver iniciar su vuelo a los hermanos Wright también pudo ver a Armstrong posar su planta sobre la luna, el XXI, donde la tecnología nos rodea en todas y cada una de nuestras actividades cotidianas, el salto es tan acelerado que vivimos tan inmersos en él de forma que ni tan siquiera se tiene la percepción de que exista como tal, a menos que logremos encontrar uno de esos pocos momentos de paz y tranquilidad que nos hurta el rápido devenir de los acontecimientos para dedicarlo a la reflexión. Consideremos que en nuestra sociedad la proporción de usuarios de telefonía móvil (movilnautas) es tres veces mayor que la de los usuarios de Internet 
(internautas) y cinco veces mayor que la de televidentes: una sociedad donde casi la mitad de la gente utiliza mensajes de texto en sus relaciones sentimentales (García \& Fabila, 2014).

Niños y adultos generan una dependencia cuasi total en su día a día hacia las TIC, siendo impulsados por el viento del cambio de nuestra sociedad, hoy sostenida gracias a grandes bases de datos en todos los aspectos de la vida cotidiana, de la sanidad a la justicia, pasando por la educación. Aunque no todos los miembros de nuestra sociedad están conectados a la red (recordemos el concepto de "analfabetos funcionales" que deviene hoy en "analfabetos tecnológicos") por falta de recursos o por carencia educativa (Guerrero Cárdenas, 2016), en el caso de los niños, el efecto mimético con su entorno, con sus compañeros, basta para considerar que el número de ajenos absolutos a las TIC es nulo o casi nulo. Viven, vivimos, insertos en una cultura de lo visual comunicándonos con imágenes, con hipertexto. Esto nos provoca nuevas formas de articular nuestro pensamiento a partir de nuevas lógicas cognitivas. Y si eso sucede con adultos que se formaron al margen de las TIC, entendamos que este discurso hipermedia en el momento de desarrollo intelectual, incide en la creación de nuevas formas de asociación multidimensional, no secuenciales, de saberes, cuyos enlaces facilitan el acceso a las ventanas de conocimiento como consecuencia de la virtualización del proceso de lectura y escritura (Caballero, 2009). Esto para algunos indica que desde los diferentes contextos educativos deberemos marcarnos como una de las principales metas a conseguir en la educación favorecer que los datos, la información y el conocimiento constituyan una base sólida sobre la que asentar todos los procesos reflexivos y todos los procesos de toma de decisiones con el fin de mejorar la calidad de todos los aspectos de la vida tomando esa información global, distribuida por los diferentes medios (Aznar, Cáceres \& Hinojo, 2005).

Llegado a este punto aparece siempre una sombra: no solo podemos y debemos contemplar el lado dulce, que nos facilita la vida y nos permite importantes ahorros de tiempo y energía, sino que hay que prevenir el lado agrio, con los riesgos y amenazas, sean físicas e incluso psicológicas. Tomemos por ejemplo, esa desconexión mental que se da en una persona cuando está presente físicamente en un lugar, pero su atención se centra en alguien ausente, lo que provoca que esté más pendiente del teléfono que de los presentes. Este efecto que puede parecer al tiempo trivial, pero por otra parte realmente distorsionador del proceso de aprendizaje, aparece rodeado de muchos otros riesgos que, de una u otra forma, con mayor o menor intensidad, pueden afectar a todas y cada una de las etapas del desarrollo humano, como la nomofobia (miedo irracional a salir de casa sin un teléfono móvil), tinnitus (generación de un zumbido en el oído que causa molestias y disminuye la capacidad de escuchar), adicciones tecnológicas, incidencia en trastornos alimenticios y todo ello sin hablar de asuntos mucho más graves como el ciberbullying y el sexting, elementos que pueden llegar a abocar a los niños al suicidio (García \& Fabila, 2014; García-Pina, 2008; Bringué \& Sádaba, 2009). 


\section{El docente ante el reto}

La velocidad a la que las TIC evolucionan y con ello transforman o al menos matizan nuestra sociedad no suele verse reflejada en la formación docente, que requiere de una planificación holística, consensuada, centrada en la ética y de forma especial en los valores educativos, con exigencias acordes a la gran responsabilidad y compromiso que el ejercicio de sus funciones precisa, en consonancia con la dinámica educativa actual (García, Gutiérrez, Mújica \& Henríquez, 2016) no circunscribiéndose al conocimiento y manejo de tecnologías y sus usos como herramientas aplicadas al aprendizaje, sino proponiendo paradigmas, destrezas y competencias más profundas. (Guerrero, 2015). Hay notables experiencias (Delgado, Mengual, López \& Vázquez, 2015; Rayón, 2015; Pérez-Escoda, Castro-Zubizarreta \& Fandos, 2016; Valdivieso \& González, 2016) en este sentido. Experiencias que cubren numerosos experimentos metodológicos, se han introducido herramientas TIC en la docencia ordinaria y en ocasiones se ha peleado duramente para evitar problemas que parecían asociados inexcusablemente, como el plagio (Reche, Quintero \& Marín, 2016). Baste recordar que existen colegios que piden a los niños que presenten los trabajos escritos a mano y no impresos para asegurarse de que al menos una vez estos se lean.

Una vez más, es imposible soslayar aspectos negativos del medio. Si tomamos al teléfono como referencia, al ser un dispositivo pequeño fácil de esconder para su uso en el aula, incluso si este se prohíbe, vemos que durante el transcurso de una clase representa un instrumento que abre un nuevo frente al posibilitar trampas durante los exámenes, además de ser un elemento distractor, existiendo estudios que relacionan la frecuencia de uso del teléfono con el fracaso escolar. Y no es un único problema: consideremos que la mensajería (del SMS al WhatsApp) provoca cambios en su forma de redacción, eliminando vocales y distorsionando las frases, lo que ha sido una fuente de preocupación en ocasiones (García \& Fabila, 2014). Mucho más graves son comportamientos o conductas inadecuadas, deshonestas o carentes de valores éticos o morales que los niños y niñas llevan a cabo y otros factores negativos como el antecitado "facilitación del plagio" (Reche, Quintero \& Marín, 2016). No obstante, ese gran potencial educativo que poseen las tecnologías en el aula, al que se suman las posibilidades que tienen para el desarrollo cognitivo del niño, provoca que los orillemos.

\section{El niño ante el dispositivo: conocimiento del mismo}

Partimos de una realidad constatable en nuestro día a día: no hace falta que enseñemos a los alumnos a usar sus dispositivos, muchas veces incluso será al revés y ellos podrán enseñarle trucos al docente 
sobre los mismos. Los hijos de quienes para poder hacer los deberes en casa tenían que consultar la biblioteca paterna y, caso de que esta se quedara corta debían acudir a una biblioteca pública donde poder transcribir en su cuaderno los hallazgos que encontraran o, con suerte, poder fotocopiar algunas páginas de libros allí encontrados, no siguen ese mismo camino; ellos tienen la mayor de las bibliotecas a su alcance, a "tiro de clic". La inmensa, aunque no siempre correcta, Wikipedia aparece omnipresente, junto con otras páginas, algunas de gran altura (por ejemplo la hemeroteca nacional) y otras menos ortodoxas donde pueden encontrarse trabajos escolares clasificados por materias y cursos.

Es una senda muy hollada que debemos aprovechar para encontrar en ellas nuevas maneras de generar conocimiento y saberes en todas sus formas y contextos con una forma de comunicación mucho más dinámica (Guerrero, 2015), para sacar el jugo a esa comunicación que se establece de forma natural entre ellos usando estos canales para llegar a un nuevo aprendizaje. Así (Bringué \& Sádaba, 2009), podemos distinguir dos formas de aprendizaje junto a los compañeros: una, basada en la amistad, que les lleva a desarrollar normas compartidas a la hora de publicar información y otra que les lleve de forma natural a nuevas formas de comunicación más especializadas, además de permitir incrementar el contacto entre alumnos, profesores y padres, o como herramienta organizativa.

\section{Marco legal}

El cauce por el que pretende discurrir la experiencia propuesta en el presente artículo es el marcado por el Real Decreto 126/2014, de 28 de febrero, por el que se establece el currículo básico de la Educación Primaria (BOE, 2014).

Dentro del decreto se establece como una de las siete competencias del currículo la competencia digital, sobre la que se pone especial énfasis en la utilización de las TIC para buscar, seleccionar información y presentar conclusiones, aludiendo de manera clara y concisa a las destrezas y habilidades propias de la alfabetización informacional. Otra de las competencias, la quinta, relativa a competencias sociales y cívicas con alguna asignatura específica como "Valores sociales y cívicos" con criterios de evaluación tales como "Emplear las nuevas tecnologías desarrollando valores sociales y cívicos en entornos seguros", debe emparejarse con la anterior para nuestros propósitos.

Veamos una breve relación de los puntos que son de interés a la hora de circunscribir el presente trabajo a los límites del Real Decreto.

- Listado de las competencias del currículo: artículo 2.2, página 19352. En concreto la tercera (Competencia digital) y la quinta (Competencias sociales y cívicas). Observemos que el legislador remarca "Para una adquisición eficaz de las competencias y su integración efectiva en el currículo, 
deberán diseñarse actividades de aprendizaje integradas que permitan al alumnado avanzar hacia los resultados de aprendizaje de más de una competencia al mismo tiempo."

- Elementos transversales: artículo 10, página 19356. Subraya la necesidad de emplear en todas las asignaturas las Tecnologías de la Información y la Comunicación y la educación cívica, entre otras. Abunda en sus puntos 2 y 3 en el interés de la formación en valores.

- Área de Ciencias de la Naturaleza, página 19367: el bloque de iniciación a la actividad científica tiene como criterios de evaluación "obtener información relevante sobre hechos o fenómenos previamente delimitados, haciendo predicciones sobre sucesos naturales, integrando datos de observación directa e indirecta a partir de la consulta de fuentes directa e indirectas y comunicando los resultados".

- Área de Ciencias Sociales, página19374: en el bloque de contenidos comunes se recoge "utilizar las tecnologías de la información y la comunicación para obtener información, aprender y expresar contenidos sobre Ciencias Sociales".

- Área de Lengua Castellana y Literatura, página 19382: entre los criterios de evaluación del bloque comunicación escrita: leer, se especifica que el alumnado tiene que demostrar saber "utilizar las TIC de modo eficiente y responsable para la búsqueda y tratamiento de la información".

- Área de Matemáticas, página 19388: en el bloque de procesos, métodos y actitudes en matemáticas, uno de los criterios de evaluación se indica "Utilizar los medios tecnológicos de modo habitual en el proceso de aprendizaje, buscando, analizando y seleccionando información relevante en Internet o en otras fuentes, elaborando documentos propios, haciendo exposiciones y argumentaciones de los mismos".

- Área de Educación Artística, página 19402: en el bloque educación audiovisual, entre sus criterios está "utilizar las tecnologías de la información y la comunicación de manera responsable para la búsqueda, creación y difusión de imágenes fijas y en movimiento".

- Área de Educación Artística, página 19403: en el bloque expresión artística, aparece "Utilizar recursos bibliográficos, de los medios de comunicación y de internet para obtener información que le sirva para planificar y organizar los procesos creativos, así como para conocer e intercambiar informaciones con otros alumnos".

- Área de Educación física, página 19409: considera que el alumnado debe saber "extraer y elaborar información relacionada con temas de interés en la etapa, y compartirla, utilizando fuentes de información determinadas y haciendo uso de las tecnologías de la información y la comunicación como recurso de apoyo al área". 


\section{Acercamiento a la ética del estudiante infantil}

Pero ¿y el verdadero protagonista? Subamos la apuesta y metamos en el juego a la ética, que a fin de cuentas es lo que nos ocupa. ¿Consideramos capaz al niño de usar las TIC dentro o fuera del aula y al tiempo hacer una valoración ética? Existen investigaciones que sostienen que los niños pequeños pueden interactuar en forma polémica, dar opiniones y fundamentarlas, al menos con un argumento, incluyendo en el proceso otras voces en el discurso (yo creo, otros opinan que..., etc.) (Ortega, 2016).

Existen distintos enfoques, desde los más generales, por ejemplo cuando Aznar et al. (2005), enumeran elementos como la vulnerabilidad y la limitación personal y colectiva, como sustento de la interdependencia; el beneficio y mejoramiento de las condiciones de vida de los demás, como condición necesaria de nuestro propio bienestar; el valor de la identidad y la cultura ajenas, como fuente de afirmación de nuestra propia identidad y cultura; la responsabilidad de preservar el patrimonio de las generaciones futuras, como condición de supervivencia de la especie; el respeto a la dignidad y los derechos de la persona, como sustento de la solidaridad y como garantía de la estabilidad social, a los más específicos (véase por ejemplo Ramos (2013) al referenciar dos ejes: la experimentación de la ciudadanía digital y la comprensión de los derechos de autor). Nuestra propuesta, como se verá, es más elemental, más simple, reduciéndose a un pequeño apartado de ese punto tan global que resulta la "experimentación de la ciudadanía digital".

Para el menor, la pantalla, sea de su teléfono si lo tiene ya, de una tableta o similar - si parece exagerado que demos esto por cotidiano, tal vez baste acudir a las noticias de los periódicos para encontrarnos con titulares como "El 40\% de los menores de dos años accede regularmente a tabletas o móviles. El uso de la tecnología llega al 90\% en el caso de niños de entre 10 y 15 años" (El Periódico, 2015)- es algo más que para sus padres, que muchas veces la ven en sus manos como una forma de vigilancia hacia sus hijos (García-Piña, 2008), un cordón umbilical que los ata a los padres. Es una ventana por la que ven el mundo... y por la que se dan a conocer.

La propuesta actual pasa por pretender enfrentar al alumno al espejo oscuro de la pantalla apagada. A su propio reflejo, más allá de la tecnología de la información. No por despojar a la realidad de la informática, cosa ya del todo imposible en nuestra sociedad, sino para que el brillo de la tecnología no les oculte los efectos de la misma. Y es que a través de esa nueva linterna mágica no solo reciben, polarizada, la imagen del mundo exterior, sino que también retransmiten la suya. Así, según Lipoetsky \& Serroy (2009), surge una serie de preguntas tales como:

¿qué efectos tiene esta proliferación de pantallas en nuestra relación con el mundo y con los demás, con nuestro cuerpo y nuestras sensaciones? ¿Qué clase de vida cultural y democrática anuncia el triunfo 
de las imágenes digitalizadas? (...) ¿Hasta qué punto reorganiza este despliegue de pantallas la vida del

Bajo este punto de partida, empleamos el símil del espejo negro, esa nueva realidad del uso de las pantallas como espejos virtuales, de forma que tanto permiten la ocultación como la transparencia y a través de las cuales no solo vemos cómo se comportan los demás, sino que percibimos una imagen distorsionada de cómo lo hacemos nosotros, pues no podemos olvidar que muchas cosas que podamos considerar "naturales" lo son por venirnos así dadas por la cultura en la que estamos insertos, cultura que evoluciona y a gran velocidad, por lo que si esta cultura puede cambiar, lo que en un momento dado reputamos como bueno también se vería afectado. Aún más: hoy nos parecen normales cosas que hace un par de décadas podían sonar a disparate para la inmensa mayoría, como considerar más cercanos emocionalmente a los amigos virtuales que parecen ofrecer un amor a distancia antes que a la familia real que te rodea, pero con la que no te comunica una pantalla de plasma, a pesar de que la cercanía parezca prometer un mayor grado de confidencia.

Ante esto ¿qué pasa si apagamos la pantalla, si dejamos al ser humano enfrentado a su reflejo en el espejo oscuro de su móvil, de su tableta apagado, enfrentado a él mismo y solo a él? Según Lipovetsky y Serroy (2009) en el adulto aparece el miedo. La sobreinformación. El hecho de que tenemos nuestras limitaciones y somos incapaces de discernir qué es lo verdaderamente importante cuando nos inundan con millones de datos. Pero un niño que absorbe como una esponja todo lo que le dan... muchas veces sin reflexionar o discernir sobre lo dado, no posee ese temor. El peligro que apuntan los autores es previsible, y la solución que apuntan es la necesidad de la "pantalla asistida": la colaboración de los docentes como herramienta imprescindible para poder crear ese marco intelectual preciso que impida que se muera ahogado en el aluvión de información.

Otro peligro apuntado es la deificación de la red (solo lo que en ella está existe, y además todo lo que en ella aparece es cierto) representa por otra parte que puede quebrar el vínculo social, nos comunicamos sin conocernos. $\mathrm{O}$, con sus palabras, "el universo hipermoderno de la pantalla o el mundo sensible en proceso avanzado de desrealización".

Al margen de esa infosaturación que apuntábamos, también hay que prever otros riesgos o, dicho de otra forma, hay que anticiparle al niño el peligro de que se dé para evitarlo. Por ejemplo, podríamos hablar de problemas con la propia percepción, engañarse a sí mismo o creer que tenemos un cuerpo distinto al que tenemos. Puede ir del clásico complejo de Adonis, donde nos vemos más bellos de lo que en realidad somos, a las muchas tipologías de dismorfofobia, que provocan que, ante el espejo (el espejo de plasma en este caso) siempre nos veamos más feos de lo que somos. O, además de esta distorsión física, su paralela mental, que podemos llamar "el yo del espejo", encajando en esta categoría cambios de personalidad: gente apocada, que en la red aparece como muy activa, cuando 
no extrovertida o incluso violenta, o al revés, personas que de sí son muy abiertas, pero que en foros virtuales permanecen calladas.

\section{Conclusiones}

Las TIC contribuyen, siguiendo a Caballero (2009), a la consolidación de grupos sociales como práctica social en sí misma, pues la tecnología es un producto socio-cultural. Por lo tanto, la incorporación de las TIC y sus contenidos digitalizados al ámbito educativo, posibilita nuevos sistemas de comunicación y, en consecuencia, deviene en una práctica social emergente, a espacios de comunicación deslocalizados. Generalmente, los colonizarán los alumnos por sí mismos. E trabajo en el aula se centraría en enfrentar al alumnado al espejo oscuro de su pantalla apagada.

Las reflexiones obtenidas pueden servirles de herramienta para vencer los riesgos que las TIC conllevan. Puede parecer a priori que por nacer rodeados de pantallas aprenderán antes a cuidarse de ellos, pero podemos afirmar que no es así. Para ello, basta que nos fijemos en que los primeros nativos digitales ya están cursando estudios universitarios y no solo no muestran en apariencia un manejo más diestro de las herramientas TIC que sus predecesores una década atrás, sino que aparece una ya no sospechosa, porque ha sido confirmada (Díaz, 2014), ingenuidad digital que les hace mantener la guardia baja ante los riesgos de la red. Eso les lleva a, por ejemplo, no proteger sus datos personales, dando un punto de interés para este trabajo desde las primeras etapas de la educación.

Cabe cerrar acudiendo a un clásico, Weizenbaum, que ya en 1978 decía que el profesor de informática debe tener el valor de resistir a la tentación de ser arrogante, y enseñar principalmente mediante el ejemplo, la validez y la legitimidad de un conocimiento más conciliador. No hay mejor manera de desnudar el pensar de los alumnos que empezar por uno mismo (Weizenbaum, 1978).

\section{References}

Aznar Díaz, I., Cáceres Reche, M. P., \& Hinojo Lucena, F. J. (2005). El impacto de las TICs en la sociedad del milenio: nuevas exigencias de los sistemas educativos ante la "alfabetización tecnológica". Eticanet, 2(4), 177-190.

BOE (2014). Real Decreto 126/2014, de 28 de febrero, por el que se establece el currículo básico de la Educación Primaria. BOE de 1 de marzo de 2014. Madrid: Boletín Oficial del Estado Recuperado (01/03/2017) de https://www.boe.es/boe/dias/2014/03/01/pdfs/BOE-A-2014-2222.pdf

Bringué Sala, X. \& Sádaba Chalezquer, C. (2009). La generación interactiva en España. Niños y adolescentes ante las pantallas. Madrid: Ariel. 
Busnell, D. D. (1967). The Computer in American Education. New York: John Wiley \& Sons

Caballero, S. L. (2009). Tránsito digital en el ámbito educativo. Revista Iberoamericana de Educación, $(48 / 6) .1-13$.

Campos Roselló, F. J. (1985). La informática a examen. Valencia: Tirant Lo Blanc

Delgado Algarra, E. J., Mengual Andrés, S. López Meneses, E., \& Vázquez-Cano, E. (2015). La justicia evaluativa en la valoración de proyectos grupales: propuesta y aplicación en el Grado de Educación Primaria. Pulso. Revista de Educación, 38, 163-177.

Díaz Sáenz, J. R. (2014). Factores críticos en la adopción de medidas de seguridad utilizadas por los alumnos de los Centros formativos universitarios de tecnologías TIC al usar herramientas 2.0. Tesis doctoral. Valencia: UPV.

El Periódico (2015). El 40\% de los menores de dos años accede regularmente a tabletas o móviles. Barcelona: Zeta. Recuperado (01/03/2017) de http://www.elperiodico.com/es/noticias/tecnologia/ los-menores-dos-anos-accede-regularmente-tabletas-moviles-4226851

García García, B., Gutiérrez Hidalgo, C., Mújica de López, M., \& Henríquez García, M. A. (2016). Paradojas, contrastes y aproximación ética en el uso de las TIC desde la Educación Superior. Revista de Estudios y Experiencias en Educación, 15(29), 29-48. doi: https://doi.org/10.21703/rexe.20162929482

García Martínez, V., \& Fabila Echauri, A. M. (2014). Nomofilia vs. Nomofobia, irrupción del teléfono móvil en las dimensiones de vida de los jóvenes. Un tema pendiente para los estudios en comunicación. Razón y Palabra, 18(86).

García-Peñalvo, F. J. (2016). Proyecto TACCLE3 - Coding. En F. J. García-Peñalvo \& J. A. Mendes (Eds.), XVIII Simposio Internacional de Informática Educativa, SIIE 2016 (pp. 187-189). Salamanca, España: Ediciones Universidad de Salamanca.

García-Peñalvo, F. J., \& Kearney, N. A. (2016). Networked youth research for empowerment in digital society. The WYRED project. In F. J. García-Peñalvo (Ed.), Proceedings of the Fourth International Conference on Technological Ecosystems for Enhancing Multiculturality (TEEM'16) (Salamanca, Spain, November 2-4, 2016) (pp. 3-9). New York, NY, USA: ACM. doi: https://doi.org/10.1145/3012430.3012489 García-Peñalvo, F. J., Reimann, D., Tuul, M., Rees, A., \& Jormanainen, I. (2016). An overview of the most relevant literature on coding and computational thinking with emphasis on the relevant issues for teachers. Belgium. doi: https://doi.org/10.5281/zenodo.165123

García-Piña, C. A. (2008). Riesgos del uso de internet por niños y adolescentes. Estrategias de 
seguridad. Acta Pediátrica de México, 29(5), 273-279.

Guerrero Cárdenas, E. (2016). Educación líquida y TIC. Dialéctica. Revista de Investigación Educación, 12(1), 64-77.

Lipovetsky, G., \& Serroy, J. (2009). La pantalla global. Barcelona: Anagrama.

Moreno Alejo, J. A. (2016). Utilización de un Videojuego como Recurso Didáctico Apoyado en las TIC para fomentar los Valores Éticos en los Estudiantes del Grado Noveno del Ciclo de Educación Básica del Instituto Técnico Industrial de Villavicencio en Colombia. Tesis de Maestría. Universidad TecVirtual. Recuperado (01/03/2017) de http://hdl.handle.net/11285/619666

Nora, S., \& Minc, A. (1980). Informe Nora Minc. La informatización de la sociedad. Madrid: Fondo de cultura económica

Oltra Gutiérrez, J. V. (2014). Los menores como consumidores. La pequeña empresa ante el fenómeno de las redes sociales y su uso por menores. En A. Domingo Moratalla (Ed.), Juventud y Redes Sociales (pp. 261-290). Madrid: OJE.

Ortega de Hocevar, S. (2016). Nuestros niños argumentan. Traslaciones. Revista latinoamericana de lectura y escritura, 3(6), 47-77.

Pérez-Escoda, A., Castro-Zubizarreta, A., \& Fandos, M. (2016). La competencia digital de la Generación Z: claves para su introducción curricular en la Educación Primaria. Comunicar. Revista Científica de Educomunicación, 24(49), 71-80. doi: http://dx.doi.org/10.3916/C49-2016-07

Prensky, M. (2001). Digital Natives, Digital Immigrants Part 1. On the Horizon, 9(5), 1-6. doi: http:// dx.doi.org/10.1108/10748120110424816

Ramos Ahijado, S. (2013). Educación musical en primaria y en secundaria a través de Wikipedia como herramienta de aprendizaje en la docencia. MID. Memorias de Innovación Docente, 2012-2013. Universidad de Salamanca. Recuperado (01/03/2017) de http://hdl.handle.net/10366/122626

Rayón Rumayor, L. (2015). Guía docente Interculturalidad y recursos tecnológicos, Grado en Magisterio de Educación Primaria. Curso Académico 2016-17 $4^{\circ}$ curso $1^{\circ}$ Cuatrimestre Universidad de Alcalá. Recuperado (01/03/2017) de https://www1.uah.es/estudios/asignaturas/programas/ G430/430044_G430_2015-16.pdf

Reche Urbano, E., Quintero Ordoñez, B., \& Marín Díaz, V. (2016). De la competencia informacional al ciberplagio en Educación Primaria. Revista científica electrónica de Educación y Comunicación en la Sociedad del Conocimiento, 2(16), 263-281. 
Seoane Pardo, A. M. (2016). Computational thinking beyond STEM: an introduction to "moral machines" and programming decision making in Ethics classroom. In F. J. García-Peñalvo (Ed.), Proceedings of the Fourth International Conference on Technological Ecosystems for Enhancing Multiculturality (TEEM'16) (Salamanca, Spain, November 2-4, 2016) (pp. 37-44). New York, NY, USA: ACM. doi: http://dx.doi. org/10.1145/3012430.3012494

Valdivieso Guerrero, T. S., \& González Galán, M. A. (2016). Competencia Digital Docente: ¿Dónde estamos? Perfil del docente de educación primaria y secundaria. El caso de Ecuador. Pixel-Bit. Revista de Medios y Educación, 49, 57-73. doi: http://dx.doi.org/10.12795/pixelbit.2016.i49.04

Weizenbaum, J. (1978). La frontera entre el ordenador y la mente. Madrid: Pirámide. 